\title{
Detector characterization in GEO 600
}

\author{
A M Sintes ${ }^{1,5}$, P Aufmuth ${ }^{2}$, C Aulbert $^{5}, \mathbf{S}$ Babak $^{6}, \mathbf{R}$ Balasubramanian $^{6}$, \\ B W Barr ${ }^{3}$, S Berukoff ${ }^{5}$, S Borger ${ }^{5}$, G Cagnoli $^{3}$, C A Cantley ${ }^{3}$, \\ M M Casey ${ }^{3}$, S Chelkowski $^{2}$, D Churches ${ }^{6}, \mathrm{C} \mathrm{N} \mathrm{Colacino}^{2}$, \\ D R M Crooks ${ }^{3}, C_{\text {Cutler }}^{5}$, K Danzmann $^{2,4}$, R Davies ${ }^{6}$, R Dupuis ${ }^{3}$,

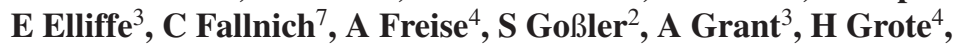

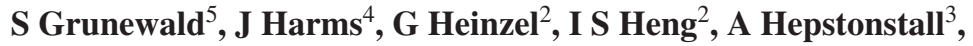 \\ M Heurs ${ }^{2}$, M Hewitson ${ }^{3}$, J Hough ${ }^{3}$, R Ingley ${ }^{8}$, Y Itoh ${ }^{5}$, O Jennrich ${ }^{3}$, \\ R Jones ${ }^{3}$, S Hutter ${ }^{3}$, K Kawabe K $^{4}$ C Killow ${ }^{3}$, K Kötter ${ }^{2}$, B Krishnan ${ }^{5}$, \\ V Leonhardt ${ }^{2}$, H Lück ${ }^{2,4}$, B Machenschalk ${ }^{5}$, M Malec ${ }^{2}$, C Messenger $^{8}$,

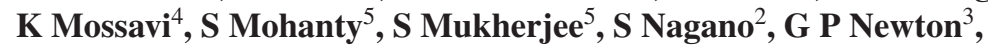 \\ M A Papa ${ }^{5}$, M Perreur-Lloyd ${ }^{3}$, M Pitkin $^{3}$, M V Plissi ${ }^{3}$, V Quetschke $^{2}$, \\ S Reid ${ }^{3}$, L Ribichini ${ }^{4}$, D I Robertson ${ }^{3}$, N A Robertson ${ }^{3}$, S Rowan ${ }^{3}$,

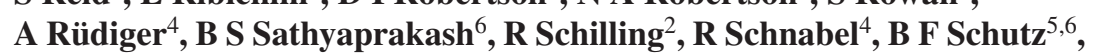 \\ F Seifert ${ }^{4}$, J Smith ${ }^{4}$, P Sneddon ${ }^{3}$, K A Strain ${ }^{3}$, I Taylor ${ }^{6}$, C I Torrie ${ }^{3}$, \\ A Vecchio ${ }^{8}$, H Ward $^{3}$, U Weiland ${ }^{2}, \mathbf{H}$ Welling $^{7}$, P Williams $^{5}$, B Willke $^{2,4}$, \\ W Winkler ${ }^{4}$, G Woan ${ }^{3}$ and I Zawischa ${ }^{7}$

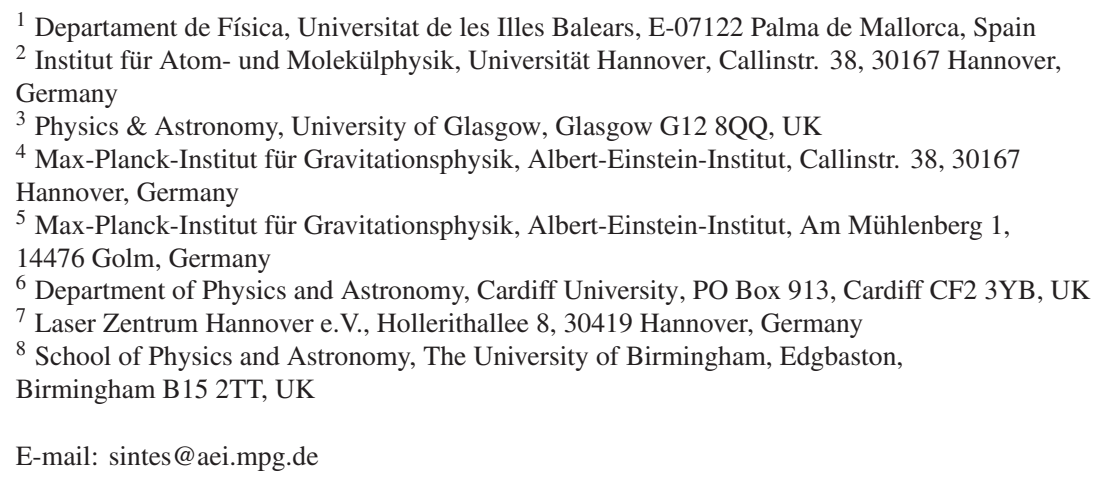

Received 30 June 2003

Published 11 August 2003

Online at stacks.iop.org/CQG/20/S731

\begin{abstract}
The GEO 600 interferometric gravitational wave detector conducted its first science run (S1) from 23 August 2002 to 9 September 2002. The GEO 600 data acquisition system is described together with some software tools developed for doing detector characterization and data analysis. Detector characterization results are also being presented.
\end{abstract}

PACS numbers: $04.80 . \mathrm{Nn}, 95.55 . \mathrm{Ym}$, 93.65.+e 


\section{Introduction}

The detector characterization of the gravitational wave detector GEO 600 [1-3] is a collaborative work of scientists from different groups joined in the GEODC group [4]. GEODC brings together both experimentalists and theorists. This is important since a detailed knowledge of the detector and also expertise on data analysis are required.

The detector characterization of GEO 600 has two main purposes. The first purpose is to understand and characterize the GEO 600 gravitational wave detector, e.g., identify noise sources, misbehaviour, and study noise propagation, so that experimentalists can improve the detector and bring it to the design sensitivity. The second purpose is to give support to the different astrophysical data analysis searches, e.g., providing vetoes for data analysis.

The members of the GEODC group are involved in data acquisition, data transfer and storage; the development of software tools to facilitate data access and data analysis, including on-line monitors; development of new algorithms and the analysis itself of the data from the GEO 600 detector and auxiliary channels. GEODC members participate also in the LIGO Scientific Collaboration (LSC) upper-limits working groups [5]. These are the inspiral [6], burst [7], periodic [8] and stochastic [9] working groups. The purpose of these groups is to develop code and analyse LIGO and GEO 600 data to search for gravitational wave signals.

During the year 2002, GEO 600 performed several engineering and science runs, some of them in coincidence with LIGO and TAMA, e.g., E7 (28 December 2001-14 January 2002) and S1 (23 August 2002-9 September 2002), with duty cycles of 75\% and 98\%, respectively [10]. These data have been analysed by the GEODC group by means of software tools that have been developed, in particular the GEO-Tools and GEO++. Attention is focused mainly on data quality studies, the development of veto strategies and the understanding of the interferometer behaviour.

The rest of the paper is organized as follows: section 2 describes the GEO 600 data acquisition system. In section 3, several software tools developed for detector characterization are presented, the GEO on-line detector characterization system is discussed in section 4 . Finally in section 5, we present some detector characterization analysis results.

\section{Data acquisition and data transfer}

GEO 600 requires a sophisticated data acquisition system (DAQS) able to acquire, store and

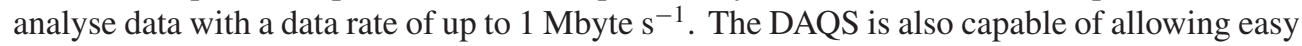
access to both stored and real-time data [11]. An overview of the components of the DAQS is given in figure 1.

The data are acquired by analogue-to-digital-converter boards installed in three data collecting units. They are located at the central building and the two end stations. The maximum sampling rates correspond to $16384 \mathrm{~Hz}$ and $512 \mathrm{~Hz}$, and each channel can be decimated to lower rates. In the central building 32 fast channels and 64 slow channels are available, and in each of the end buildings we can use 16 fast channels. Most of these channels are being used for detector characterization only. Environmental data are recorded in all of them while detector data are only acquired in the central station. Environmental data include data from magnetic field sensors, seismic sensors and microphones, monitors that record wind speed and direction, temperature, air pressure, vacuum pressure inside the tanks and also the supply voltage. Detector data include data from the laser system, the two mode-cleaner cavities and the Michelson interferometer.

All these data, together with information coming from the LabView system, that controls the position and the alignment of the optical components in GEO 600, and the detector status 


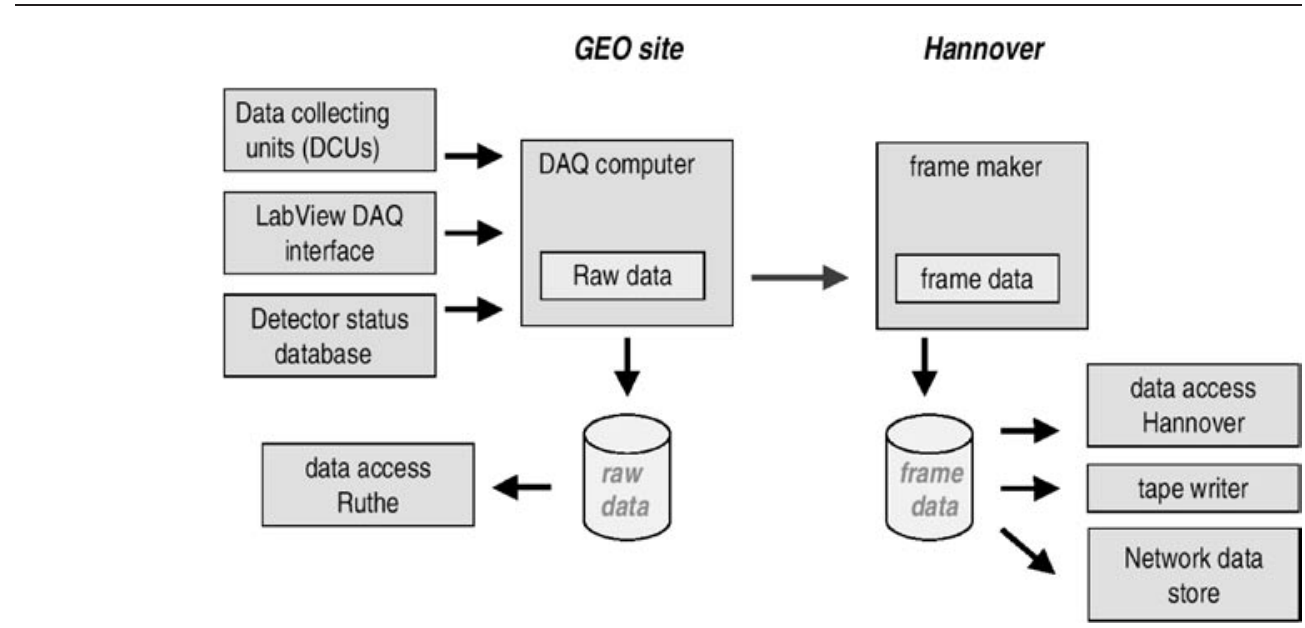

Figure 1. Overview of the components of the data acquisition system.

database, are then processed by the data-collector that is a software tool that writes the data into a GEO internal format. The data are temporarily stored on a disk array at the detector site and then sent via a radio link to Hannover. At Hannover, the data are accessed by the frame-maker that immediately converts them into the so-called frame format, a common data format for all laser interferometric detectors.

The data are also distributed to the other groups, in particular, the data are written onto tapes or pushed via the network to Cardiff and Golm, where larger storage facilities are available. In these three locations frame servers are running providing data to the entire GEO 600 collaboration on request. File naming convention has been unified with other interferometric detectors in order to facilitate data exchange and analysis. Trend data and a calibrated $h(t)$ channel are produced and stored using the same frame format.

In order to give a feeling of the volume of data, only during the S1-run, more than a terabyte of data were acquired, transferred, stored and analysed [10].

Data analysis is mainly performed on several Beowulf clusters. These are located at Hannover, Golm, Birmingham and Cardiff. While the latter three are dedicated to astrophysical analysis, the Hannover cluster is in charge of performing the first time-critical data analysis. This includes running the on-line detector characterization monitors that are described in section 4 .

\section{Software tools}

In order to facilitate data access and data analysis, several software tools have been developed and are being used by the GEO 600 collaboration. These include different libraries, programs and scripts for detector characterization and analysis of GEO 600 data, under some common software environments, and several databases containing all sort of information, from the detector status information to the data analysis results. A brief description follows for some of them.

There is a restricted web page set up, the so-called GEO info page, providing an interface to several databases and tools, e.g., an environmental conditions monitor, a time conversion tool, a data-viewer and the interferometer controls. The databases include the detector status database and the frequencies database. The detector status database contains detailed information regarding the status of the different detector subsystems and the data acquisition system, 
including descriptions of the signals acquired and calibration information. The frequencies database contains information regarding modelled and measured resonances of the GEO 600 detector, along with the frequencies that were found in the various recorded data channels. Both databases allow easy access to relevant information necessary for the understanding of the detector and the analysis of the data.

Very useful for doing detector characterization and data analysis of GEO 600 data are the GEO-Tools. The GEO-Tools are a collection of libraries, scripts, Matlab mex files and $\mathrm{m}_{-}$files. The GEO-Tools are open source and can be downloaded from http://www.astro.gla.ac.uk/users/hewitson/downloads.html. Documentation is provided with the source code together with examples that illustrate the possible uses. Among others these include: a frame server written in $\mathrm{C}$ to serve frame data to various clients; a client designed to connect to the server, retrieve a subset of the available channels and write new frame files on the local disk; commands to retrieve all sorts of information from the frame server or local frame files, e.g., a channel list, available calibration, lock status information and any channel data for any particular time; a frame viewer as a set of Matlab scripts files that provide a GUI interface to frame files; C-code and mex files for creating and using filters and window functions; time conversion tools.

Given the massive data analysis problem posed by the interferometric detectors, there is a need to develop software capable of handling efficiently large amounts of data. The GEO++ digital signal processing library [12] is a software centred around $\mathrm{C}++$ classes which provides the necessary framework to carry out GEO 600 data analysis and detector characterization tasks. The primary aim of GEO++ is to provide an environment where potentially a large quantity of data can be filtered through data analysis or detector characterization pipelines in real time. More explicitly, GEO++ provides the basic tools of analysis such as data containers, filters, transforms as well as software modules which enable the analysis to be carried over networked workstations (a Beowulf cluster) using MPI. GEO++ provides a frame file interface for GEO 600 and LIGO data and also a systematic way of recording the output of the analysis in the form of MySQL database records and frame files. The software is designed for later expansion in the form of detector characterization monitors and astrophysical search modules. GEO++ comes with a base monitor and search classes which developers of data analysis or detector characterization tasks can use to code their favourite algorithms. The source code and documentation are available at http://www.astro.cf.ac.uk/pub/R.Balasubramanian/geo++/index.htm.

Another project under development is the detector characterization robot (DCR). The aim of DCR is to construct a reliable and efficient automated system that is able to keep track of all change points in all relevant channels. The output information will be stored in a database in order to support future data mining tasks. The emphasis here is placed on controlling the false alarm rate, i.e., in controlling and understanding the quality of the data stored in the database. A description of the different aspects of the DCR project and its implementation in GEO 600 can be found in $[13,14]$.

For all these different software tools sophisticated algorithms are being developed [15-17].

\section{GEO 600 on-line detector characterization system}

GEO 600 on-line detector characterization system (GODCS) is based on GEO++ which offers a flexible scheme to develop and integrate monitors into a pipeline and run several monitors at a time.

The basic idea is that monitors can be simple and small modules and we can get complex functionality by connecting them together into pipelines. Monitor developers do not need to 
be bothered about the data flow, how the data are read from the frame files, nor details of database output. A monitor is limited to processing a single chunk of data and preserves a state between consecutive data chunks. In addition, since all the filtering of the data is done within GEO++, this simplifies the job of monitor writers and prevents errors in the monitor codes. Examples of monitors would be power spectral density estimators, line removal tools, transient detectors, correlators etc. A description on how to write a monitor can be found in [18], and in [19] the reader can find how to run a GODCS pipeline. GODCS can run in two modes: single processor or multiprocessor using MPI.

One particularity of GODCS is that it has the capacity of chaining together monitors, each monitor analysing data modified by the previous monitors. This is very useful if we want to develop monitors which act only when another monitor triggers an event.

Here there is a partial list of the detector characterization monitors available: PQMon, PsdMon, SaturationMon, PowerTrackerMon, LockStatusMon, InspiralMon, GlitchMon, GlitchInPowerLineMon, ExcessPowerMon, CorrelationMon, RemoveLinesMon, TFClusterMon, FrameChannelSummaryMon.

A job description file could look something like the one below (see [19] for details):

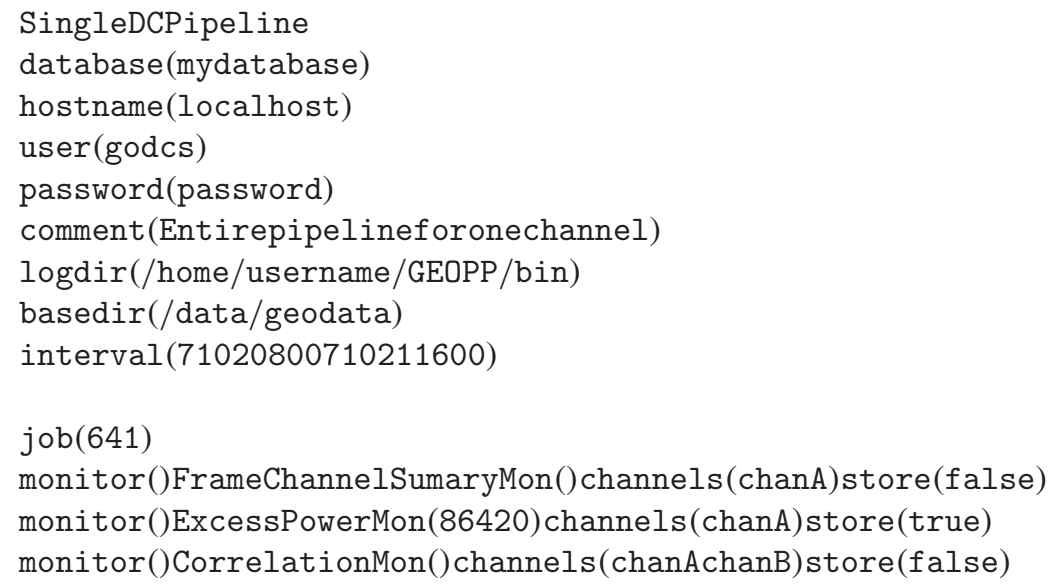

GODCS has been extensively used for detector characterization purposes and also in the context of burst and inspiral searches in the S1 data from GEO 600.

\section{Data analysis}

In this section we briefly describe the kind of analysis that is being performed on the GEO 600 data for detector characterization purposes.

The first issue is to check the quality of the acquired data in the different channels. This is important, not only for characterizing the detector itself, but also because the auxiliary channels could be useful as vetoes when analysing the data for gravitational wave signals. Therefore, we need to ensure that the values of the acquired data are reasonable, so that we can extract from them the most meaningful information possible. We need to determine the frequency band in which an actual signal is recorded and the amount of signal above the DAQS noise level. It is also important to check the calibration functions and the channel behaviour.

A different question is the calibration of the $h(t)$ channel from the detector output. During the S1 run, the configuration of GEO 600 was of a power recycled interferometer. The calibration was performed by means of injecting calibration peaks at particular frequencies. An extension of the DAQS software performed the calculations necessary to calibrate the 

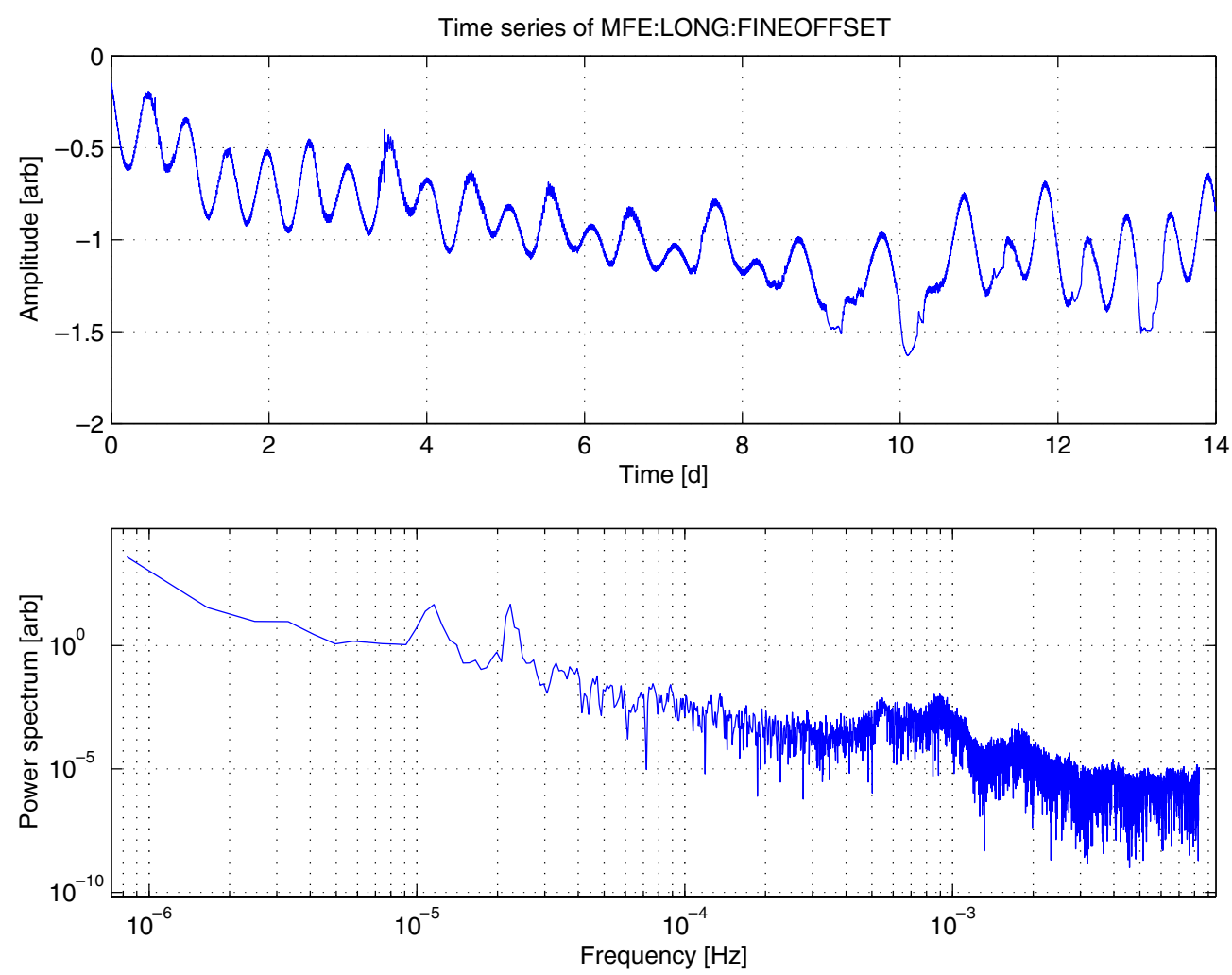

Figure 2. (Top) The graph shows the Michelson differential longitudinal feedback in lock which is applied at the far east end mirror (channel MFE:LONG:FINEOFFSET) over a time of 14 days starting on 24 August 2002 at 0:00 UTC. The sampling rate is 1/60 Hz. (Bottom) Power spectral density of the above time series. The $24 \mathrm{~h}$ related peak around $11.6 \mu \mathrm{Hz}$ is mainly originated by the daily temperature fluctuations while the one at $22.4 \mu \mathrm{Hz}$ corresponding to $12 \mathrm{~h} 24 \mathrm{~min}$ is due to tidal effects.

detector continuously. More complicated is now the case of the dual-recycled interferometer. A detailed description of the calibration scheme for GEO 600 can be found in [20].

Several investigations have been carried out using S1 and test data. Among others, we are interested in the study of the long term performance of the interferometer. As an example, we can clearly observe tidal effects and daily temperature fluctuations causing differential armlength changes, as shown in figure 2, and how they affect the optical gain. Plenty of valuable information can easily be obtained analysing the trend data generated by the DAQS.

Recognizing lines in the amplitude spectral density of the different data channels, and measuring their characteristics may give us one of the basics for detector characterization. With this aim, an automated line detection code was developed and used [17]. It is based on a robust algorithm, using running median and interquartile ranges, able to detect the various spices of lines: ultra-thin lines, thin lines or broad lines, which can appear isolated or crowded together. Using this code, we analysed several channels and detected hundreds of lines, which were collected on a MySQL database (the frequencies database) available on the web. 


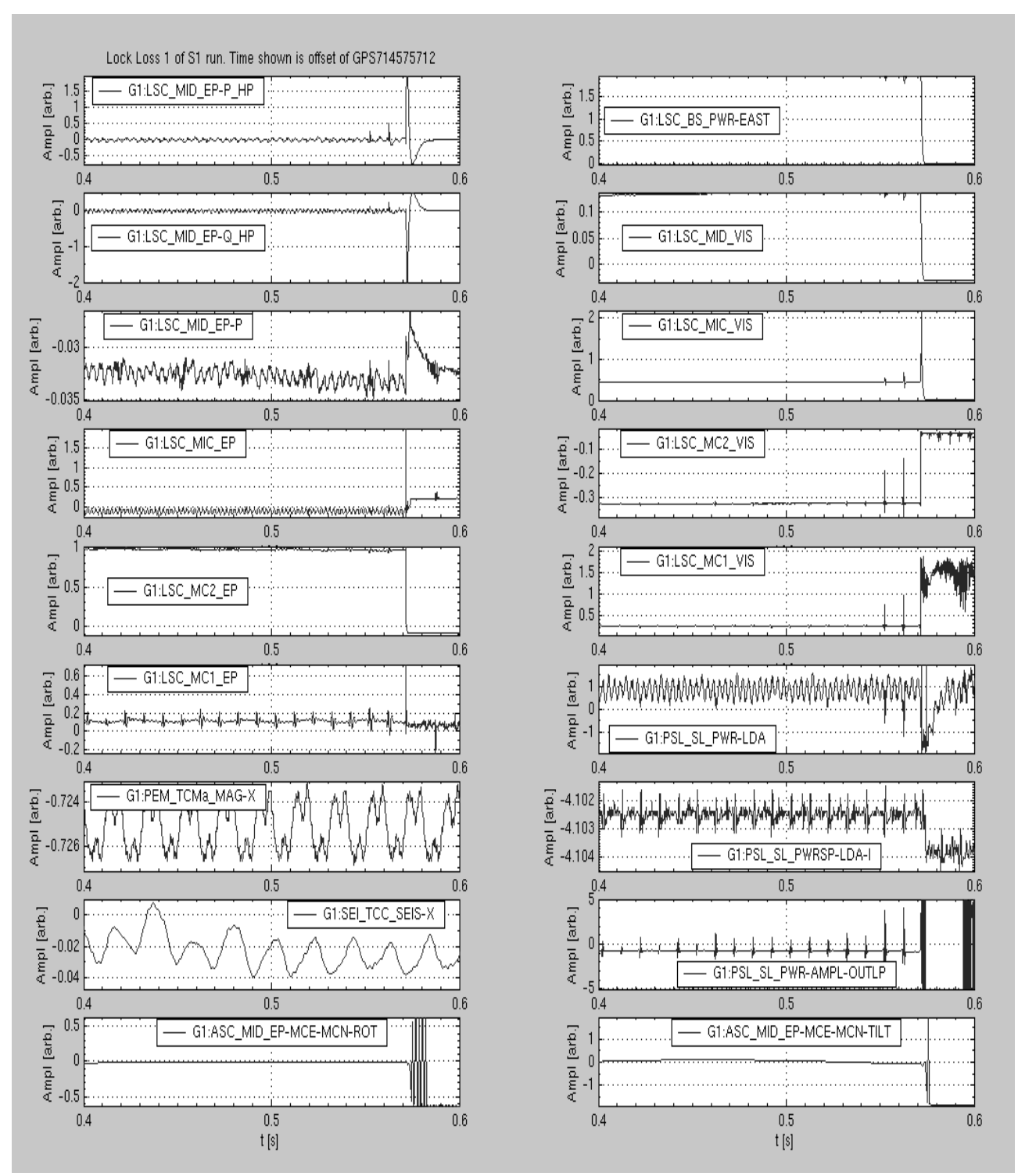

Figure 3. Snapshot of the relevant channels during the first lock loss of the S1 run. This lock loss was due to a growing spike sequence of exactly $10 \mathrm{~ms}$ in the laser power G1:PSL_SL_PWRAMPL-OUTLP (second graph from bottom on the right-hand side). The last two spikes before loss of lock are coupling into a few other channels.

In order to improve the detector performance, we need to investigate lock losses and understand their likely causes. During S1, the GEO 600 interferometer operated in a very stable mode with a duty cycle of about $98 \%$. The first lock lasted over five days. In this case the lost lock was due to a series of laser spikes, as shown in figure 3. Two monitors implemented in GEO++ became very useful: one for detecting glitches in the data (GlitchMon) and another for recording times when the lock status channels changed their value (LockStatusMon). With the help of these two monitors the different losses of lock were identified and investigated. 


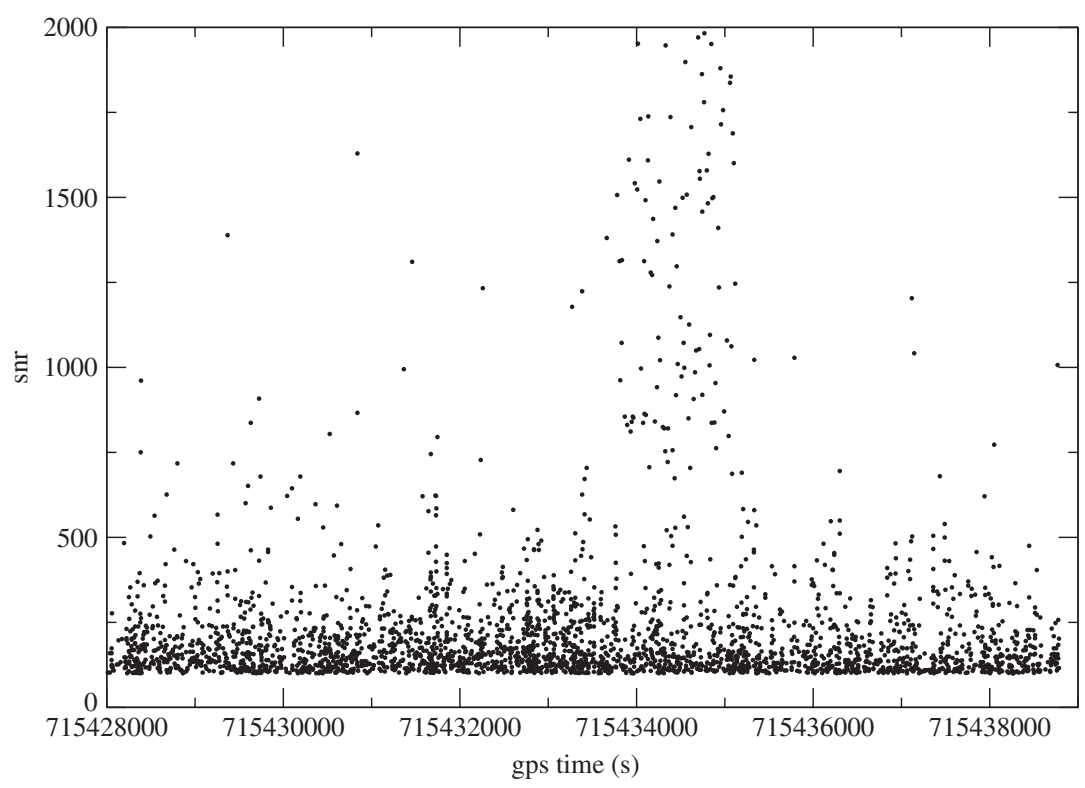

Figure 4. Scatter plot of SNR versus GPS time of the events for GEO++ TFClusterMon. The centre of the graph shows an excess of events and this corresponds to the signal injection period.

The majority of the lock losses took place during the daily maintenance period due to some kind of operator intervention. Other causes were, e.g., a van passing next to the east end building, and a strong earthquake in Sicily, Italy (on 9 September 2002 at 1:21:27 UTC, magnitude 6.1 and with a travel time of 3 min $44 \mathrm{~s}$ ).

The full GEO-S1 data were analysed by means of the GEO++ monitors, running both on-line and off-line. But also, lots of test were carried out on a section of $3 \mathrm{~h}$ of data chosen as playground data for initial analysis. Investigations performed included the study of glitches and excess power in different channels and their coupling into $h(t)$. In particular, seismic, main-power, laser and interferometer channels were carefully investigated with the aim of identifying well-understood veto channels. Although many of them did not seem to give convincing vetoes, in some cases possibly because of electrical pickup contamination that was later amended, we could use the high power detector error point in phase and quadrature (channels G1:LSC_MID_EP-P_HP and G1:LSC_MID_EP-Q_HP), leading towards the P/Q veto and the design of the PQMon [21]. The rationale behind the $\mathrm{P} / \mathrm{Q}$ veto is that any candidate gravitational wave burst that is in G1:LSC_MID_EP-P_HP should also appear in G1:LSC_MID_EP-Q_HP in the correct ratio. If a glitch detected in G1:LSC_MID_EP-P_HP does not show up in the correct ratio (within limits) in G1:LSC_MID_EP-Q_HP it cannot be a gravitational wave signal, hence providing a veto.

Another monitor extensively used is the TFClusterMon. This monitor identifies regions in a spectrogram where excess power is observed. This monitor is suitable for detecting many kind of transients, including burst and inspiral signals. Using TFClusterMon, we could clearly identify the times in which inspiral injections took place during S1 (see figure 4), which were confirmed a posteriori using matched filtering. TFClusterMon also found periodicities in the occurrence of events in $h(t)$ that are still under investigation.

Other extensive GEO data analysis studies have been carried out in the context of pulsar, stochastic, inspiral and burst searches and will be reported elsewhere. 


\section{Acknowledgments}

The authors would like to thank the Particle Physics and Astronomy Research Council in the UK (PPARC), the German Bundesministerium für Bildung und Forschung (BMBF) and Land Niedersachsen (the State of Lower Saxony, Germany) for their funding and continuous support of the GEO 600 project. AMS also thanks the Spanish DGICYT Research Project no BFM 2001-0988 for financial support.

\section{References}

[1] Danzmann K et al 1994 GEO 600-Proposal for a 600 m laser-interferometric gravitational wave antenna (MPQ Garching)

[2] Willke B et al 2002 The GEO 600 gravitational wave detector Class. Quantum Grav. 191377

[3] Webpage http://www.geo600.uni-hannover.de

[4] Webpage http://www.aei.mpg.de/sintes/GEO_DC

[5] Webpage http://www.ligo.org

[6] Webpage http://www.lsc-group.phys.uwm.edu/iulgroup

[7] Webpage http://www.ligo.caltech.edu/ /ajw/bursts/bursts.html

[8] Webpage http://www.lsc-group.phys.uwm.edu/pulgroup

[9] Webpage http://www.phys.utb.edu/stochastic

[10] Hewitson M et al 2003 Proc. 7th Gravitational Wave Data Analysis Workshop (Kyoto, 2002) Class. Quantum Grav. $20 \mathrm{~S} 581$

[11] Kötter K et al 2002 Data acquisition and detector characterization of GEO 600 Class. Quantum Grav. 191399

[12] Babak S et al 2002 GEO++ digital signal processing library GEO Technical Document Webpage http:// www.astro.cf.ac.uk/pub/R.Balasubramanian/geo++/index.htm

[13] Mohanty S D and Mukherjee S 2002 Towards a data and detector characterization robot for gravitational wave detectors Class. Quantum Grav. 191471

[14] Webpage http://www.aei.mpg.de/ /mohanty/DCR/DCRindex.html

[15] Mohanty S D 2002 Median based line tracker (MBLT): model independent and transient preserving line removal from interferometric data Class. Quantum Grav. 191513

[16] Mukherjee S 2003 Proc. 7th Gravitational Wave Data Analysis Workshop (Kyoto, 2002) Class. Quantum Grav. $20 \mathrm{~S} 925$

[17] Babak S, Borger S, Itoh Y, Mohanty S and Sintes A M 2002 Auto line detection GEO Technical Document

[18] Balasubramanian R 2002 GODCS monitor writing guide GEO Technical Document

[19] Balasubramanian R 2002 GODCS users manual GEO Technical Document

[20] Hewitson M et al 2003 Proc. 7th Gravitational Wave Data Analysis Workshop (Kyoto, 2002) Class. Quantum Grav. $20 \mathrm{~S} 885$

[21] Kötter K et al 2003 Proc. 7th Gravitational Wave Data Analysis Workshop (Kyoto, 2002) Class. Quantum Grav. $20 \mathrm{~S} 895$ 